\title{
PREVALENCIA DE SOBREPESO Y OBESIDAD, CONSUMO DE ALIMENTOS Y PATRÓN DE ACTIVIDAD FÍSICA EN UNA POBLACIÓN DE NIÑOS ESCOLARES DE LA CIUDAD DE BOGOTÁ
}

\author{
ESPERANZA FAJARDO BONILLA, ND. M.SC ${ }^{1 *}$ \\ Luis Alberto Ángel ArAngo, M.D \\ ${ }^{1}$ Grupo de Investigación CATCH, Facultad de Medicina, Universidad Militar Nueva Granada, Bogotá. \\ ${ }^{2}$ Profesor Titular, Facultad de Medicina, Universidad Nacional de Colombia.
}

\begin{abstract}
Resumen
Las cifras de sobrepeso y obesidad infantil se han incrementado en los últimos años en Colombia, factor que resulta alarmante teniendo en cuenta la relación que existe entre estos problemas nutricionales y el desarrollo de enfermedades crónicas en etapas posteriores de la vida. Con miras al diseño de estrategias de prevención y control del desequilibrio nutricional en la infancia, este trabajo tiene como objetivo determinar la prevalencia de sobrepeso y obesidad y analizar el consumo de alimentos y el patrón de actividad física en un grupo de niños escolares. Se estudiaron 326 niños entre 7 y 11 años de edad, de $2^{\circ}$ a $5^{\circ}$ de primaria de dos colegios de la ciudad de Bogotá. Se tomaron medidas antropométricas y a partir del peso y la talla se construyó el índice de masa corporal para la clasificación nutricional y se relacionó con los indicadores de adiposidad que se establecieron a partir de la relación entre los pliegues cutáneos y perímetros corporales e impedancia bioeléctrica. Para evaluar el consumo de alimentos se utilizó el método de recordatorio de 24 horas y el cuestionario de frecuencia de consumo y se aplicó una encuesta sobre los hábitos de actividad física. Los resultados demuestran que el $22.4 \%$ de este grupo de estudiantes presentan sobrepeso y $15,3 \%$ obesidad. Se observó una relación directa entre el índice de masa corporal y el porcentaje de grasa hallado a partir de la impedancia bioeléctrica y los pliegues cutáneos:a medida que aumenta el índice de masa corporal, aumenta el porcentaje de grasa corporal. El consumo de energía y nutrientes no mostró diferencia estadísticamente significativa entre los estudiantes con un estado nutricional normal de los que presentan sobrepeso y obesidad al relacionarlos por género. El patrón de actividad física en los niños se caracteriza por actividades sedentarias como ver televisión o juegos de video. La presencia de sobrepeso y obesidad está asociada con las medidas de la circunferencia de la cintura $(\mathrm{p}=0.022)$ y el porcentaje de grasa determinado por el método de impedancia bioeléctrica ( $\mathrm{p}=0.001)$. En el grupo estudiado se observa alta prevalencia de sobrepeso y obesidad, distribución abdominal de la grasa corporal principalmente en los hombres lo que se encuentra asociado a poca actividad física vigorosa.
\end{abstract}

Palabras clave: Obesidad infantil, actividad física, alimentación.

* Correspondencia: Esperanza Fajardo Bonilla - esperanza.fajardo@unimilitar.edu.co. Dirección: Tr. 3 № 49-00, Facultad de Medicina, Universidad Militar Nueva Granada, Teléfono: 6500000, Bogotá, Colombia. 


\title{
PREVALENCE OF OVERWEIGHT AND OBESITY, FOODS INTAKE AND PHYSICAL ACTIVITY PATTERN IN A POPULATION OF SCHOOL CHILDREN IN THE CITY OF BOGOTÁ
}

\begin{abstract}
Figures of infant overweight and obesity have increased over the past years in Colombia, posing an alarming issue when taking into account the relationship between these nutritional problems and the development of chronic diseases in later stages of life. In order to design prevention strategies and control of unbalanced nutrition in infancy, this study aimed to determine the overweight and obesity prevalence and to analyze the food intake and physical activity pattern in a group of school children. Three hundred twenty six children aged between 7 and 11 years, 2 nd and 5 th graders from two schools of Bogotá were examined. Anthropometric measures were taken and from weight and height, the body mass index was built for nutritional classification and was related with the adiposity indicators established from the relationship between skinfold, body perimeter and bioelectric impedance. In order to evaluate the foods intake, the 24-hour reminder method and the intake frequency questionnaire were used and a survey over the physical activity behaviors was conducted. The results show that $22.4 \%$ of this group is overweight and $15.3 \%$ is obese. A direct relationship between body mass index and fat percentage found with bioelectric impedance and skinfolds was observed: As the body mass index increases the percentage of body fat also increases. The energy and nutrients intake did not show statistically significant difference between students with normal nutritional status and those overweight and obese when they were related by gender. The physical activity pattern in children is characterized by sedentary activities such as watching TV or play video games. The presence of overweight and obesity is associated with measurements of waist circumference $(p=0.022)$ and fat percentage determined by bioelectric impedance method $(p=0.001)$. In the studied group, a high prevalence of overweight and obesity was observed as well as abdominal distribution of body fat mostly in males which is associated with poor vigorous physical activity.
\end{abstract}

Key words: Infant obesity, physical activity, feeding.

\section{PREVALÊNCIA DE OBESIDADE, CONSUMO DE ALIMENTOS E PADRÃO DE ATIVIDADE FÍSICA EM UMA POPULAÇÃO DE CRIANÇAS EM IDADE ESCOLAR NA CIDADE DE BOGOTÁ}

\begin{abstract}
Resumo
Os números mostram que nos últimos anos a obesidade infantil aumentou na Colômbia, fator que é alarmante, visto que a relação existente entre estes problemas nutricionais e o desenvolvimento de doenças crônicas nas etapas posteriores da vida. Com vistas ao desenho de estratégias de prevenção $e$ controle do desequilíbrio nutricional na infância, este trabalho tem como objetivo determinar a prevalência de sobrepeso e obesidade e analisar o consumo de alimentos e o padrão de atividade física em um grupo de crianças em idade escolar. Foram estudadas 326 crianças entre 7 e 11 anos de idade, de $2^{\circ}$ a $5^{\circ}$ ano da educação básica de dois colégios da cidade de Bogotá. Foram tomadas medidas antropométricas e a partir do peso e da estatura foi construído o índice de massa corporal para a classificação nutricional e relacionou-se com os indicadores de adiposidade que foram estabelecidos a partir da relação entre as dobras cutâneas e perímetros corporais e impedância bioelétrica. Para avaliar o consumo de alimentos foi utilizado o método de aviso de 24 horas e o questionário de frequência de consumo e foi aplicado um questionário sobre os hábitos de atividade física. Os resultados demonstram que $22.4 \%$ deste grupo de estudantes apresentam estão acima do peso e 15,3\% obesidade. Observou-se
\end{abstract}




\begin{abstract}
uma relação direta entre o índice de massa corporal e a porcentagem de gordura encontrada a partir da impedância bioelétrica $e$ as dobras cutâneas: A medida que aumenta o índice de massa corporal, aumenta a porcentagem de gordura corporal. $\mathrm{O}$ consumo de energia e nutrientes não mostrou diferença estatisticamente significativa entre os estudantes com um estado nutricional normal dos que estão acima do peso ou estão obesos ao relacioná-los por gênero. O padrão de atividade física nas crianças se caracteriza por atividades sedentárias como assistir televisão ou jogar videogames. A presença de obesidade está associada com as medidas da circunferência da cintura $(\mathrm{p}=0.022)$ e a porcentagem de gordura determinada pelo método de impedância bioelétrica ( $\mathrm{p}=0.001)$. No grupo estudado observa-se alta prevalência de obesidade, distribuição abdominal da gordura corporal principalmente nos homens o que encontra-se associado a pouca atividade física vigorosa.
\end{abstract}

Palavras chave: Obesidade infantil, atividade física, alimentação.

\section{Introducción}

El estado nutricional del individuo es el resultado del balance entre las necesidades de energía, nutrientes esenciales y su gasto. Los factores físicos, genéticos, biológicos, culturales, psicológicos, sociales, económicos y ambientales, pueden dar lugar a una ingestión insuficiente o excesiva de nutrientes, o a impedir la utilización óptima de los alimentos consumidos. Por otra parte, los patrones de alimentación, específicamente relacionados con el exceso de peso combinados con el sedentarismo, son aspectos reconocidos por la Organización Mundial de la Salud (OMS) como factores de riesgo para el desarrollo de enfermedades cardiovasculares que representan la primera causa de muerte a nivel mundial $(1,2)$.

La OMS considera que las escuelas son fundamentales para el desarrollo de hábitos de vida saludables y la realización de programas de prevención, al reconocer que la edad escolar es un periodo importante para promover y consolidar habilidades en todas las áreas del desarrollo, para reafirmar hábitos de alimentación saludables y para que los niños y las niñas alcancen una excelente calidad de vida y así garanticen las reservas necesarias para cubrir el gasto de energía que demandan las nuevas actividades asumidas durante esta época de la vida. La nutrición adecuada y el establecimiento de conductas saludables en los niños puede contribuir, no sólo a prevenir, problemas de salud inmediatos y a promover un estilo de vida sano, sino a reducir el riesgo de que el niño desarrolle alteraciones crónicas, como obesidad, diabetes tipo 2 , enfermedad cardiovascular, o una combinación de éstas, en etapas posteriores de la vida (3).
En Colombia, ese doble problema nutricional que se está afrontando actualmente, entre la desnutrición y la obesidad, exige establecer prioridades y estrategias de acción en el campo de la salud pública, agregando a las políticas dirigidas a superar el hambre y la desnutrición, la prevención y el control del sobrepeso y la obesidad, y por ende, la presencia de enfermedades crónicas. A las acciones integrales que involucren educación sobre alimentación y nutrición, se deben adicionar entornos favorables para la actividad física y disfrute del tiempo libre (4).

Este estudio se orientó a establecer el estado nutricional de una población escolar, la frecuencia en el consumo de alimentos y el patrón de actividad física en una población de escolares de la ciudad de Bogotá, teniendo en cuenta la metodología aceptada internacionalmente (5), y aceptando que es un punto de partida para emprender acciones de prevención de la enfermedad y promoción de la salud, fundamentadas en estrategias educativas e integrales tal como lo recomiendan organismos nacionales e internacionales de salud y más recientemente la Encuesta Nacional de la Situación Nutricional en Colombia (6).

\section{Antecedentes}

Es conocido que la adecuada alimentación en el niño escolar, mejora su calidad de vida. Específicamente, el estado nutricional y el ambiente psicosocial que enmarcan el crecimiento y desarrollo del niño influyen en su función cognoscitiva. Por lo tanto, todas las acciones que se apliquen para mejorar la nutrición de los niños, redundarán posteriormente en su salud y comportamiento, tanto en la etapa de formación, como en su desempeño social posterior como adultos. 
El conocimiento que se tenga sobre la interrelación entre nutrición y salud, tanto a nivel de la familia como de las instituciones responsables de la salud de madres y niños, será factor básico para asegurar la incorporación y el rendimiento normal del niño en la escuela y en su vida futura (7).

Los reportes sobre el estado nutricional de la población colombiana refieren avances positivos frente a la lucha contra la desnutrición, con la disminución porcentual tanto en la "desnutrición crónica" como en la "desnutrición global", aspecto interpretado como un avance en el país en cuanto a las intervenciones concretas. Sin embargo, también se muestra una tendencia creciente de la obesidad acompañada de una deficiente actividad física (6), estos últimos aspectos, señalados desde épocas anteriores en los reportes a nivel internacional según los datos de países en vías de desarrollo. Desde 1995 se reportó que en Colombia, el 2.6\% de los niños preescolares presentaban sobrepeso (8).

Por otra parte, la Organización Mundial de la Salud ha señalado que la enfermedad cardiovascular, considerada como la primera causa de muerte en el mundo, causará tres veces más muertes e inhabilidad en Suramérica y el Caribe que las enfermedades infecciosas y los sistemas de la salud en la región no están preparados adecuadamente para ocuparse de esta enfermedad crónica (9).

La presencia de obesidad y los altos niveles de colesterol en sangre en niños, puede ser indicador de enfermedad cardiovascular en etapas posteriores de la vida, incluso si los factores de riesgo se mejoran durante la juventud, por lo tanto, orientar desde la niñez los esfuerzos para la prevención de estos factores de riesgo, a través de programas de intervención eficaces, puede tener un efecto positivo en el estado de salud de la persona en etapas posteriores $(10,11)$. Las intervenciones para la salud basadas en el comportamiento, han sido las más acertadas para la prevención de riesgo cardiovascular, también se ha demostrado, un incremento en la actividad física y la adopción de mejores hábitos alimentarios, en niños y adolescentes $(12,13)$. La Organización Mundial de la Salud, hace un llamado a intervenir para reducir los riesgos de enfermedad y promover una vida sana y señala que centrarse en los riesgos para la salud es fundamental para la misma intervención, lo cual redunda en gran beneficio para la salud pública (14).
El papel de la nutrición humana en la salud de las poblaciones constituye hoy en día una de las grandes áreas de la investigación y de las políticas sanitarias para contribuir al bienestar de las personas tanto en el ámbito individual como social. En la medida en que cobran interés las enfermedades crónicas como causa principal de mortalidad, la identificación y control de los factores nutricionales asociados a estas enfermedades, cobran interés, como en el caso de las alteraciones cardiovasculares (2).

Conocer cuál es la situación nutricional de una población es fundamental para las distintas intervenciones en materia de salud pública. En el desarrollo del estudio de la nutrición y la alimentación se han identificado diferentes métodos para hacer tal diagnóstico, dentro de los cuales se encuentra la antropometría, la impedancia bioeléctrica, la evaluación del consumo de alimentos y por ende el consumo de energía y nutrientes. Los datos del estado nutricional y del patrón de actividad física, pueden orientar programas que van desde la educación hasta la definición de políticas concretas para las poblaciones dada la estrecha relación entre alimentación, ejercicio físico y salud $(1,15)$.

El cambio más importante en la composición corporal asociado a la obesidad es el aumento de peso. Se ha cuestionado el uso del índice de masa corporal en los niños y en los adolescentes al considerar sus constantes variaciones en la composición corporal por el crecimiento y la maduración. Además, la relación entre el índice de masa corporal y la cantidad de tejido adiposo cambia con la edad, el sexo y la raza, pero a pesar de estos aspectos se ha observado en diferentes estudios, que el índice de masa corporal, es más preciso que otro tipo de relaciones del peso para la estatura (16). La obesidad en los niños se define como un índice de masa corporal en el percentil 95 o más mientras que el aumento del riesgo a sobrepeso se define como un índice de masa corporal entre los percentiles $85 y<95$. El bajo peso se define con un índice de masa corporal menor del percentil $5(2,17)$.

Al igual que en los adultos, el aumento de adiposidad en niños y adolescentes está asociado a alteraciones metabólicas y cardiovasculares. La circunferencia de la cintura ha estado asociada de manera constante a los factores de riesgo metabólico en personas de diferentes edades y es un buen elemento de correlación de la grasa subcutánea y total del tronco; puede servir como un indicador de los depósitos de grasa visceral 
aumentados en los niños obesos. Freedman y colaboradores han observado de manera constante que la grasa central, valorada mediante pliegues cutáneos del tronco y las proporciones entre pliegue cutáneo del tronco y periféricos, predicen alteraciones en los lípidos, lipoproteínas y sensibilidad a la insulina. La asociación de adiposidad central con factores de riesgo es más fuerte en niños obesos y adolescentes que en personas con peso normal y la circunferencia de cintura y cadera y sus relaciones, son buenos factores pronósticos del tejido adiposo intraabdominal $(18,19)$.

En los niños resulta útil para predecir la distribución de la grasa corporal la circunferencia de la cintura, cadera y muslo, así como las medidas de los pliegues cutáneos del tríceps y subescapular. Las circunferencias de la cintura y cadera son buenos factores pronósticos del tejido adiposo abdominal. En diversos estudios se observó que la circunferencia de la cintura, esta correlacionada con antecedentes familiares de diabetes, niveles de lipoproteínas de baja densidad, niveles de colesterol de lipoproteína de alta densidad y presión arterial alta $(19,20)$.

La impedancia bioeléctrica se considera una técnica adecuada para medir la composición del cuerpo en los niños porque las medidas se toman rápidamente, no es un método invasivo, es económico, sin dolor, y puede ser utilizada para estimar la composición del cuerpo en los individuos obesos. Washino et al. en 1999, observaron que la proporción entre colesterol total y colesterol de lipoproteínas de alta densidad fue mucho más alta con un estimado de 23 a $25 \%$ de grasa mediante bioimpedancia (21). Diversos factores pueden influir en sus mediciones, incluyendo el nivel de hidratación, la postura, la temperatura ambiental y/o de la piel, edad, género, estado atlético, estado de la composición del cuerpo y origen étnico (22-24).

Para la evaluación del consumo de alimentos es utilizado el Recordatorio de 24 horas, método que se puede aplicar mediante una entrevista directa y cuya calidad de la información recolectada depende de la memoria del sujeto entrevistado, de su capacidad de comunicación y cooperación, así como de la habilidad del entrevistador. Este método ha sido utilizado en estudios poblacionales a gran escala tales como el NHANES III y ha sido validado por comparación de los resultados obtenidos con otros métodos como el pesaje de los alimentos, las historias dietarias y marcadores biológicos (15, 25-27).
Con respecto a la actividad física, para la OMS la vida activa a lo largo del ciclo vital es una prioridad estratégica y recomienda que los niños realicen por lo menos 60 minutos de actividad física diaria $(2,4)$. Sin embargo la generalización de la conducta sedentaria y de la inactividad es un hecho favorecido por la vida moderna y reportado por diversos estudios. El Centro Nacional para la Prevención de Enfermedades Crónicas y Promoción de la Salud de Estados Unidos (CDC), ha reportado la poca actividad física en los niños, por lo que ha desarrollado iniciativas específicas tales como la campaña YMC (Youth Media Campaign) que invita a los niños a iniciar y mantener una mayor actividad física con el fin de evitar la obesidad, hipertensión, enfermedades cardiovasculares, diabetes mellitus, osteoporosis y algunos tipos de cáncer en etapas posteriores de la vida, con los consiguientes e inmensos costos sociales y de atención de salud (28-30).

El aumento de la prevalencia del sobrepeso y la obesidad en América Latina y el Caribe no deben interpretarse como signos de transición hacia el desarrollo $y$ es preciso considerar que la obesidad coexiste con déficit de micronutrientes y otras enfermedades carenciales, sobre todo en los grupos socioeconómicos más desprotegidos (31). Si bien en Colombia se considera que la situación nutricional del niño ha cambiado en los últimos tiempos gracias a las diferentes intervenciones individuales y colectivas, así como al desarrollo de acciones de seguridad alimentaria orientadas a los hogares y grupos más vulnerables, existe evidencia de incremento de las cifras de sobrepeso y obesidad por estudios a nivel regional con escolares, además de los últimos reportes de la Ensin 2010 (6, 32,33). Ésto, se considera un problema de Salud Pública porque existe amplia evidencia científica de que la obesidad en el niño es un factor de riesgo de continuar siendo obeso en la etapa adulta, incrementando los factores de riesgo para enfermedades cardiovasculares (34).

Al problema nutricional derivado del sobrepeso y la obesidad, se une el inadecuado consumo de alimentos, bajo consumo de frutas y verduras y alto consumo de alimentos ricos en energía (35), elevados índices de consumo de dulces, golosinas y gaseosas, acompañados de la costumbre de no desayunar (36). En Colombia, los niños y los jóvenes son los que más consumen alimentos de paquete (llamados "snack") y el consumo de verduras es bajo en todos los grupos de edad. Estudios regionales han demostrado un alto 
consumo de grasa y carbohidratos en niños y jóvenes de 6 a 18 años (6,37). Países como Chile y Argentina, ha reportado resultados similares con respecto al incremento en la prevalencia de la obesidad infantil, bajo consumo de frutas y verduras y aumento en el consumo de aceites y azúcares debido a golosinas, gaseosas y jugos, además de un de estilo de vida sedentaria $(29,38-40)$.

Con el fin de promover la actividad física en la etapa escolar, se han fijado unos lineamientos curriculares, en todo el país, para la educación física, la recreación y el deporte. En diferentes países se ha implementado en las instituciones educativas el atender el tiempo después de la jornada escolar, para satisfacer intereses del joven y brindar espacios para desarrollar sus potencialidades artísticas, deportivas y expresivas, exigencia que la escuela debe responder. Este tipo de programas ha aumentado a nivel mundial para prevenir la obesidad en los niños y en los adolescentes logrando resultados positivos en la adopción de hábitos de alimentación y actividad física saludables y de esta manera prevenir las enfermedades que se asocian con la obesidad y el sedentarismo (41-43).

\section{Metodología}

Se realizó un estudio observacional, descriptivo, de corte transversal.

\section{Población y muestra}

El universo estuvo conformado por 380 niños entre 7 y 11 años de edad, estudiantes de $2^{\circ}$ a $5^{\circ}$ de primaria en dos colegios públicos seleccionados por conveniencia en la ciudad de Bogotá. Ingresaron al estudio 326 escolares de ambos sexos y de quienes se tenía el consentimiento informado de sus padres.

Se excluyeron los escolares cuyos padres no autorizaron por escrito su ingreso al estudio.

\section{Procedimiento}

El estudio contó con la autorización de los directivos y la colaboración de los profesores de cada una de las instituciones educativas. Se envió a todos los padres de familia de los grados $2^{\circ}$ a $5^{\circ}$ una invitación escrita para participar en el estudio, en la cual se informó sobre el objetivo y la metodología a seguir. Adicionalmente se les envió el formato de consentimiento informado para ser diligenciado y devuelto al colegio. Sólo aquellos que fueron correctamente diligenciados participaron del estudio.

Ulteriormente se envió a los padres de familia que aprobaron el estudio, el cuestionario de Frecuencia de Consumo de Alimentos y Patrón de Actividad Física.

En las instalaciones de cada uno de los colegios, se realizó la toma de medidas antropométricas; peso, talla, pliegues cutáneos, perímetros corporales y la impedancia bioeléctrica. En el mismo momento y de forma aleatoria se realizó la entrevista a los niños sobre el consumo de alimentos teniendo como base el día anterior y utilizando el método del recordatorio de 24 horas. Durante la entrevista se utilizó vajilla de diferentes tamaños con el fin de precisar la cantidad de alimento consumida por el niño.

\section{Técnicas e instrumentos}

Para la recolección y registro de las medidas antropométricas, los datos de reactancia y resistencia, se utilizó hoja de cálculo en el programa Microsoft Office Excel 2003.

Para la toma de peso y talla se utilizó una báscula de peso corporal electrónica (Microlife WS 100, capacidad $150 \mathrm{~kg}$, precisión $\pm 1 \%+0.1 \mathrm{~kg}$ ) y tallímetro. Estas medidas se tomaron sin zapatos, sin medias, con pantaloneta y camiseta. La estatura se tomó con los pies y las rodillas juntas, talones, cara posterior de glúteos y cabeza bien adheridos al plano del tallímetro, se colocó una escuadra sobre el vértex y se leyó el valor de la talla, en centímetros.

El índice de masa corporal se construyó a partir de los datos de peso y talla (peso/talla2 expresado en $\mathrm{kg} / \mathrm{m} 2$ ). Para la clasificación nutricional, se utilizaron las tablas de índice de masa corporal por edad, para hombres y mujeres de 2 a 20 años establecidas por el Centro de Estadísticas de Salud de Estados Unidos (USA National Center for Health Statistics). Los valores de índice de masa corporal menores del percentil 5 son considerados como bajo peso; normal, entre el percentil 5 y menos del 85; sobrepeso entre el percentil 85 y menos del 95, y obesidad en el percentil 95 o más. 
Para las medidas de los pliegues cutáneos se utilizó el calibrador tipo Langer (precisión de $1 \mathrm{~mm}$ ) y para los perímetros corporales la cinta antropométrica flexible con una escala de $10 \mathrm{~mm}$ (error $1 \mathrm{~mm}$ ).

El pliegue cutáneo tricipital se midió en el punto medio entre el borde inferior del acromion y el olécranon, en la cara posterior del brazo.

El pliegue cutáneo subescapular se midió a $1 \mathrm{~cm}$ distal del pliegue oblicuo generado a la altura del ángulo inferior de la escápula, en dirección de abajo hacia arriba y de adentro hacia afuera en un ángulo de $45^{\circ}$ con el plano horizontal.

El pliegue cutáneo suprailíaco se midió en la línea media axilar por encima de la cresta ilíaca anterosuperior.

El pliegue de la pantorrilla se midió de forma vertical, sobre el punto máximo de la circunferencia de la pantorrilla derecha, con el sujeto sentado, rodilla a $90^{\circ}$ y relajación total de la pantorrilla.

La circunferencia del brazo se tomó en medio de la distancia entre el acromion y el olécranon, con el brazo relajado al lado del cuerpo.

La circunferencia de la cintura se tomó al final de una expiración normal, a un nivel intermedio entre el último arco costal y la cresta ilíaca, en la posición más estrecha del abdomen.

La circunferencia de la cadera se tomó a nivel del máximo relieve de los músculos glúteos, el sujeto parado con los pies juntos y la masa glútea completamente relajada. (5)

El porcentaje de grasa corporal se estimó a partir de los pliegues cutáneos, mediante las ecuaciones y la clasificación propuestas por Slaughter et al.

Con los resultados obtenidos se clasificó el porcentaje de grasa en muy bajo, bajo, óptimo, moderadamente alto, alto, muy alto (16).

Un Impedanciómetro bioeléctrico tetrapolar (RJL Systems Inc.), se utilizó para la impedancia, estableciendo la resistencia y reactancia corporales, a partir de los cuales se calculó el valor de grasa corporal, utilizando las ecuaciones de referencia del instrumento. Cada una de las mediciones se realizó tres veces y el promedio fue el valor aceptado. Las medidas de impedancia bioeléctrica fueron tomadas con los niños en posición supina luego de 10 minutos de reposo en camilla. Los electrodos fueron colocados en las extremidades derechas a nivel del dorso de la muñeca entre las apófisis estiloides radio-cubitales y en el dorso de la porción distal del tercer metacarpiano, en el cuello de pie en la línea que une los maléolos y en dorso de pie a nivel la porción distal del tercer metatarsiano (21).

El cuestionario de Frecuencia de Consumo de Alimentos y Patrón de Actividad Física, se construyó a partir de modelos estandarizados y utilizados en estudios previos $(13,44)$.

Para la anamnesis alimentaria se utilizó un formato teniendo en cuenta los grupos de alimentos y siguiendo la metodología para el recordatorio de 24 horas $(25,44)$.

Para el análisis del consumo de alimentos se utilizó la Tabla de Composición de Alimentos Colombianos del Instituto Colombiano de Bienestar Familiar, Hoja de Cálculo de Microsoft Excel (45).

\section{Análisis de datos}

Se realizó análisis univariado a través de tablas de distribución de frecuencias para variables cualitativas y medidas descriptivas de tendencia central, de posición y de dispersión para variables cuantitativas. El análisis bivariado y multivariado se realizó a través de tablas de contingencia, las diferencias de promedios y porcentajes utilizando pruebas paramétricas y no paramétricas, según la variable y su distribución de probabilidad, utilizando la "t" de Student, 2, análisis de varianzas, análisis de correlación (Pearson y Spearman) y regresión linear múltiple (multivariable y paso a paso). Para determinar significancia estadística, se estableció un nivel de 5\%. Para el análisis de los datos se utilizó SPSS versión 16.0 (SPSS Inc. Chicago, Illinois USA).

\section{Resultados}

De 326 estudiantes estudiados, 156 son niños y 170 niñas, con edad promedio es de 9.11 años. No se encontró diferencia estadísticamente significativa en la distribución por edad, nivel escolar ni género al comparar las dos instituciones educativas. 


\section{Características Antropométricas de la Población:}

En la Tabla 1 aparecen los datos antropométricos de la población total y por género; sólo se encontró diferencia significativa entre géneros para la circunferencia de la cintura y cadera, el índice cintura/cadera, los cuales fueron mayores en los niños y, los porcentajes de grasa corporal estimados a partir de los pliegues tricipital y pantorrilla e impedancia bioeléctrica, los cuales fueron mayores en las niñas.
Al relacionar la clasificación nutricional por índice de masa corporal y género, se observó mayor prevalencia de obesidad en los niños que en las niñas con una diferencia estadísticamente significativa $(p=0.002)$. (Tabla 2).

En la población total el índice cintura/cadera se distribuye de manera diferente según el género, siendo mayor la frecuencia de la distribución de la grasa a nivel superior del cuerpo en los niños que en las niñas $(\mathrm{p}=0.0001)$ (Tabla 3).

Tabla 1. Indicadores antropométricos en la población total y por género

\begin{tabular}{|l|c|c|c|c|}
\hline \multicolumn{1}{|c|}{ Variables } & Población total* & Niños (n=156)* & Niñas (n=170)* & p \\
\hline Peso (kg) & $31,91 \pm 7,78$ & $32,69 \pm 7,9$ & $31,2 \pm 7,5$ & 0.083 \\
\hline Talla (m) & $1,34 \pm 0,1$ & $1,35 \pm 0,08$ & $1,33 \pm 0,1$ & 0.262 \\
\hline Índice de masa corporal (kg /m2) & $17,44 \pm 2,47$ & $17,7 \pm 2,53$ & $17,18 \pm 2,39$ & 0.052 \\
\hline Perímetro braquial (cm) & $19,39 \pm 2,58$ & $19,67 \pm 2,66$ & $19,12 \pm 2,49$ & 0.051 \\
\hline Circunferencia de cintura (cm) & $61,21 \pm 7,13$ & $63,1 \pm 7,37$ & $59,45 \pm 6,45$ & 0,000 \\
\hline Circunferencia de cadera (cm) & $70,58 \pm 7,8$ & $71,57 \pm 8,45$ & $69,65 \pm 7,0$ & 0,026 \\
\hline Indice cintura/cadera & $0,87 \pm 0,08$ & $0,88 \pm 0,04$ & $0,85 \pm 0,09$ & 0,003 \\
\hline Pliegue tricipital (mm) & $12,01 \pm 3,99$ & $11,96 \pm 4,31$ & $12,05 \pm 3,67$ & 0,848 \\
\hline Pliegue subescapular (mm) & $8,59 \pm 3,26$ & $8,41 \pm 3,36$ & $8,75 \pm 3,16$ & 0,353 \\
\hline Pliegue suprailíaco (mm) & $10,71 \pm 4,94$ & $10,96 \pm 5,38$ & $10,47 \pm 4,49$ & 0,377 \\
\hline Pantorrilla (mm) & $9,95 \pm 3,39$ & $10,1 \pm 3,67$ & $9,8 \pm 3,12$ & 0,422 \\
\hline Indice subescapular/tricipital & $0,73 \pm 0,18$ & $0,71 \pm 0,16$ & $0,74 \pm 0,19$ & 0,235 \\
\hline \% Grasa corporal por tríceps y pantorrilla & $17,86 \pm 4,75$ & $17,23 \pm 5,5$ & $18,43 \pm 3,7$ & 0.012 \\
\hline \% Grasa corporal por tríceps y subescapular & $19,15 \pm 5,27$ & $19,19 \pm 6,0$ & $19,10 \pm 4,5$ & 0,888 \\
\hline \% Grasa por impedancia bioeléctrica & $23,77 \pm 5,49$ & $22,24 \pm 5,6$ & $25,17 \pm 4,9$ & 0,000 \\
\hline
\end{tabular}

* Promedio \pm Desviación estándar

Tabla 2. Clasificación nutricional de la población total por género e índice de masa corporal

\begin{tabular}{|c|c|c|c|c|c|c|}
\hline Clasificación Nutricional & Niños & Porcentaje & Niñas & Porcentaje & Total & Porcentaje \\
\hline Bajo Peso & 2 & 1,3 & 4 & 2,35 & 6 & 1,8 \\
\hline Normal & 85 & 54,5 & 112 & 65,8 & 197 & 60,4 \\
\hline Sobrepeso & 35 & 22,4 & 38 & 22,3 & 73 & 22,4 \\
\hline Obesidad & 34 & $21,7^{*}$ & 16 & 9,4 & 50 & 15,3 \\
\hline Total & 156 & 100 & 170 & 100 & 326 & 100 \\
\hline
\end{tabular}

* $(p=0.002)$ Niños vs. Niñas 
Tabla 3. Distribución de la población por género e índice cintura/cadera (ICC)

\begin{tabular}{|c|c|c|c|c|c|c|}
\hline ICC & Niños & Porcentaje & Niñas & Porcentaje & Total & Porcentaje \\
\hline$\leq 0,9$ & 99 & 63.5 & 141 & 82.9 & 240 & 73.6 \\
\hline$>0,9$ & 57 & $36.5^{*}$ & 29 & $17,1^{*}$ & 86 & 26.4 \\
\hline TOTAL & 156 & 100 & 170 & 100 & 326 & 100 \\
\hline
\end{tabular}

* $(p=0.000)$ Niños vs. Niñas

Del total de escolares, el 40,8\% presenta valores $\geq 0,76$ en la relación del pliegue subescapular y el pliegue tricipital, lo que representa una mayor frecuencia de distribución de grasa a nivel periférico del cuerpo. No se observó una diferencia estadísticamente significativa por género (Tabla 4).

Tabla 4. Distribución de la población por género según el índice entre el pliegue subescapular y el pliegue tricipital (IST)

\begin{tabular}{|c|c|c|c|c|c|c|}
\hline IST & Niños & Porcentaje & Niñas & Porcentaje & Total & Porcentaje \\
\hline$<0,76$ & 100 & 64 & 93 & 55 & 193 & 59,2 \\
\hline$\geq 0,76$ & 56 & 36 & 77 & 45 & 133 & 40,8 \\
\hline TOTAL & 156 & 100 & 170 & 100 & 326 & 100 \\
\hline & & & & & & \\
\hline
\end{tabular}

$(p=0.08)$ Niños vs. Niñas

Aunque el porcentaje de grasa corporal a partir del pliegue cutáneo del tríceps y de la pantorrilla según Slaughter es mayor en las niñas que en los niños, al estratificar por niveles el porcentaje de grasa corporal se observó una mayor frecuencia de niños que de niñas en los niveles de grasa moderadamente altos, altos y muy altos. El 37,6\% de las niñas presentaron niveles de grasa bajos o muy bajos, en tanto que ninguno de los niños presentó estos niveles de grasa corporal. Esta diferencia por género fue estadísticamente significativa (0.0001) (Gráfica 1).

El porcentaje de grasa corporal a partir del pliegue cutáneo del tríceps y el subescapular según Slaughter no mostró diferencias estadísticamente significativas por género, pero sí se observó mayor frecuencia de niños que de niñas en los niveles de grasa moderadamente altos, altos y muy altos.

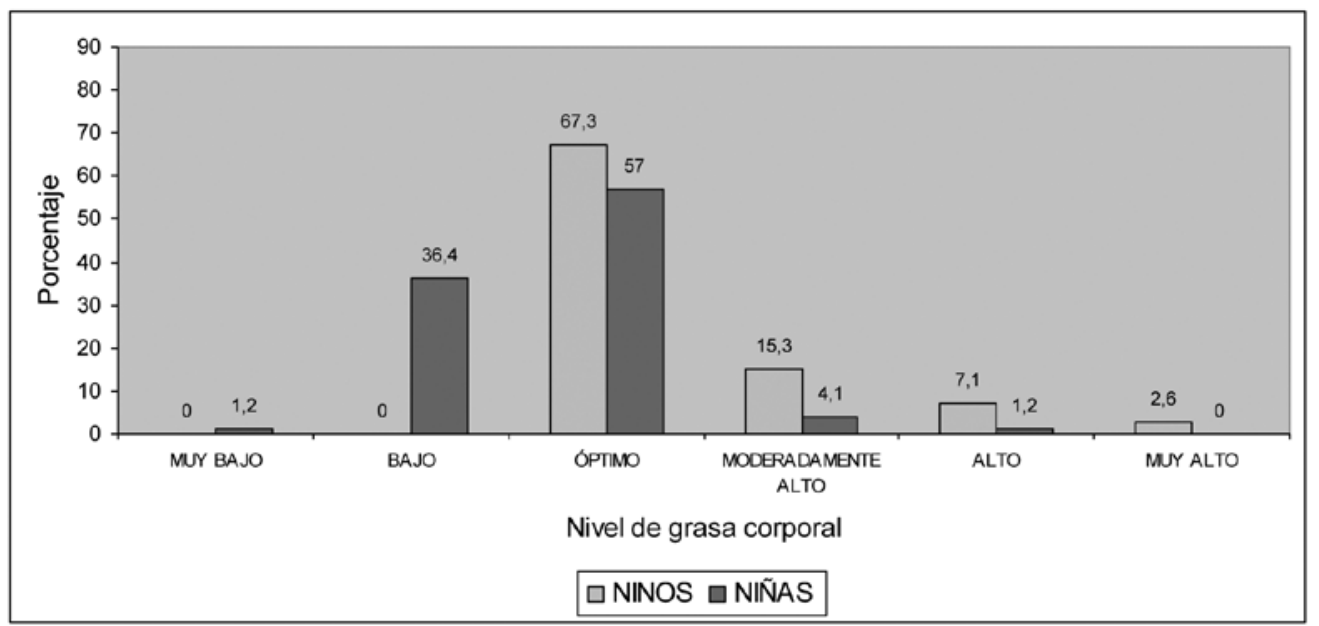

Gráfica 1. Distribución de la población según porcentaje de grasa corporal a partir de la relación del pliegue cutáneo del tríceps y la pantorrilla. 
En las niñas se observó mayor porcentaje de grasa corporal mediante impedancia bioeléctrica, diferencia estadísticamente significativa por género $(\mathrm{p}=$ 0.0001).

Al relacionar el porcentaje de grasa obtenido por impedancia bioeléctrica con la clasificación nutricional a partir del índice de masa corporal, se observa una relación lineal directa moderada entre los dos parámetros ( $\mathrm{r}=0.486 \mathrm{p}=0.000)$ : a medida que aumenta el índice de masa corporal, aumenta el porcentaje de grasa total. La misma relación se observó entre el porcentaje de grasa a partir de los pliegues cutáneos según Slaughter y la clasificación nutricional.

\section{Consumo de energía y nutrientes}

El resultado de los datos obtenidos mediante el recordatorio de 24 horas a 142 estudiantes demuestra que independiente del estado nutricional de los estudiantes, existe una diferencia en el consumo de energía $y$ de carbohidratos por género, siendo mayor en las niñas que en los niños. (Tabla 5)

En la población total el consumo promedio de proteína es del $13 \%$, grasa 35\% y carbohidratos 52\% del valor calórico total.

Tabla 5. Consumo promedio diario de energía y nutrientes en la población total

\begin{tabular}{|l|c|c|c|c|}
\hline \multicolumn{1}{|c|}{ Energía/nutriente } & $\begin{array}{c}\text { Consumo } \\
\text { en la población total* }\end{array}$ & Consumo en los niños* & Consumo en las niñas* & $\mathbf{P}^{* *}$ \\
\hline Energía (Kcal) & $1602 \pm 277$ & $1535 \pm 275$ & $1646 \pm 271$ & 0.018 \\
\hline Proteína (grs) & $54.7 \pm 14.6$ & $53.3 \pm 14.7$ & $55.7 \pm 14.5$ & 0.337 \\
\hline Grasa (grs) & $66.4 \pm 11.5$ & $64.9 \pm 10.4$ & $67.3 \pm 12.2$ & 0.234 \\
\hline Carbohidrato (grs) & $223.8 \pm 47.7$ & $211.6 \pm 43.6$ & $232 \pm 48.8$ & 0.012 \\
\hline
\end{tabular}

* Promedio \pm Desviación estándar

*** Niños vs. Niñas

\section{Patrón de actividad física}

Se establecen los datos a partir de 174 encuestas (equivalente al 55\% de los padres de familia en ambos colegios) diligenciadas por los padres de familia sobre el patrón de actividad física de sus hijos.

El mayor porcentaje de los estudiantes refiere realizar ejercicio físico 2 días a la semana por un tiempo $\geq$ 20 minutos y solamente el $17 \%$ de los 174 estudiantes, realiza ejercicio 6 a 7 días de la semana por 20 minutos o más.

La mayoría de estudiantes reciben 3 días clase de educación física en ambos colegios; no se encontró una diferencia estadísticamente significativa entre el número de días que el estudiante recibe clase de educación física y la distribución por género.

Los niños realizan ejercicio físico por más tiempo que las niñas en cada clase de educación física, realizándolo por más de 40 minutos el $67.4 \%$ de los niños contra sólo el $44.4 \%$ de las niñas ( $\mathrm{p}=0.002)$. Sólo el 31\% de los niños participan en otras actividades físicas organizadas extracurricularmente, como ir a clases de baile, gimnasia, tenis, o artes marciales sin encontrarse diferencias significativas entre ambos colegios.

En cuanto a la participación en equipos deportivos durante el último año, el 58\% de los estudiantes refiere no haber participado en equipos del colegio y el $77 \%$ manifestó no haber participado en equipos fuera del colegio. No se encontró diferencia estadísticamente significativa al relacionar esta característica por género ni por la institución educativa.

El 67.8\% de los estudiantes dedica de 1 a 4 horas diarias a ver televisión, películas de video o escuchar música durante la semana y el fin de semana se incrementa el porcentaje de estudiantes $(75.9 \%)$ que dedica el mismo tiempo a ver televisión, películas de video o escuchar música. No se observó diferencia estadísticamente significativa al relacionar el tiempo 
dedicado a la televisión, las películas o la música por género ni institución educativa durante la semana escolar ni los fines de semana.

Los niños dedican más tiempo que las niñas a los juegos de video o por computador tanto durante la semana como los fines de semana, diferencia que se encontró estadísticamente significativa $(\mathrm{p}=0.000)$ (Tabla 6).

\section{Relación entre el estado nutricional, patrón de actividad física y el consumo de alimentos}

Del total de la población el 22.3\% ( $\mathrm{n}=73)$ presenta sobrepeso y el $15.3 \%(n=50)$ obesidad, a partir del índice de masa corporal. No se observó diferencia estadísticamente significativa por institución educativa (Tabla 7).

Tabla 6. Distribución de la población por género según tiempo dedicado a videojuegos o juegos por computador.

\begin{tabular}{|c|c|c|c|c|c|c|c|c|}
\hline Tiempo (Horas/día) & \multicolumn{4}{|c|}{ Lunes - Viernes } & \multicolumn{4}{c|}{ Fines de semana } \\
\hline & Niños & Porcentaje & Niñas & Porcentaje & Niños & Porcentaje & Niñas & Porcentaje \\
\hline 0 & 23 & 31,5 & 48 & 47,5 & 15 & 20,5 & 45 & 44,5 \\
\hline$<1$ & 31 & 42,5 & 35 & 34,6 & 13 & 17,8 & 24 & 23,7 \\
\hline $1-2$ & 17 & 23,2 & 18 & 17,8 & 32 & 43,8 & 29 & 28,7 \\
\hline $3-4$ & 2 & 2,7 & 0 & 0 & 9 & 12,3 & 3 & 2,97 \\
\hline$>4$ & 0 & 0 & 0 & 0 & 4 & 5,5 & 0 & 0 \\
\hline TOTAL & 73 & 100 & 101 & 100 & 73 & 100 & 101 & 100 \\
\hline
\end{tabular}

Tabla 7. Distribución de la población por Institución según presencia de sobrepeso y obesidad por género.

\begin{tabular}{|c|c|c|c|c|c|c|}
\hline Institución & Sobrepeso & \multicolumn{2}{|c|}{ Total sobrepeso } & \multicolumn{2}{c|}{ Obesidad } & Total obesidad \\
\hline & Niños & Niñas & & Niños & Niñas & \\
\hline A & 14 & 16 & 30 & 16 & 7 & 23 \\
\hline B & 21 & 22 & 43 & 18 & 9 & 27 \\
\hline Total & 35 & 38 & 73 & 34 & 16 & 50 \\
\hline
\end{tabular}

En la población con sobrepeso y obesidad, al comparar por género la media de las medidas de los parámetros antropométricos como el peso, la talla, los pliegues cutáneos, los perímetros corporales, índices y porcentaje de grasa corporal, no se encontró una diferencia significativa en ninguno de los casos. Al comparar el grupo de estudiantes normales con los que presentan sobrepeso y obesidad, se observaron valores mayores en las medidas de peso corporal ( $p=0.03)$ en niños y niñas con exceso de peso, pliegue cutáneo tricipital (0.0001), perímetro braquial $(p=0.0001)$, circunferencia de la cintura $(p=0.0001)$, circunferencia de la cadera $(p=0.0001)$, porcentaje de grasa corporal por pliegues cutáneos $(0.0001)$ e impedancia bioeléctrica $(p=0.0001)$. No se encontró diferencia estadísticamente significativa entre la estatura de los estudiantes normales y los que presentan sobrepeso y obesidad.

El consumo de energía y nutrientes no mostró diferencia estadísticamente significativa entre los estudiantes que presentan un estado nutricional normal de los que presentan sobrepeso y obesidad al relacionarlos por género (Tabla 8). 
Tabla 8. Consumo de energía y nutrientes en la población total clasificados por índice de masa corporal como normales y con exceso de peso.

\begin{tabular}{|l|c|c|c|c|}
\hline \multicolumn{1}{|c|}{ Energía/nutriente } & \multicolumn{2}{|c|}{$\begin{array}{c}\text { Consumo en estudiantes nutricionalmente } \\
\text { normales* }\end{array}$} & \multicolumn{2}{c|}{$\begin{array}{c}\text { Consumo en estudiantes con sobrepeso } \\
\text { y obesidad* }\end{array}$} \\
\hline & Niños & Niñas & Niños & Niñas \\
\hline Energía (Kcal) & $1582.4 \pm 294.5$ & $1610.8 \pm 396.1$ & $1483 \pm 246$ & $1605 \pm 296$ \\
\hline Proteína (grs) & $56.2 \pm 15.7$ & $53.9 \pm 16.5$ & $50.0 \pm 12,9$ & $55,5 \pm 17.0$ \\
\hline Grasa (grs) & $65.2 \pm 11.8$ & $65.0 \pm 16.4$ & $64.7 \pm 8,8$ & $67.3 \pm 14.3$ \\
\hline Carbohidrato (grs) & $216.5 \pm 45.5$ & $227.6 \pm 63.0$ & $206 \pm 41,7$ & $224 \pm 52.7$ \\
\hline
\end{tabular}

* Promedio \pm Desviación estándar

Al comparar el grupo de estudiantes que presenta por el índice de masa corporal bajo peso y estado nutricional normal, con el grupo que presenta sobrepeso y obesidad, no se encontró asociación significativa entre la clasificación nutricional y el consumo de energía (OR= 0.918 IC 95\% 0.58-1.45 p=0.714), el consumo de proteínas (OR=0.791 IC 95\% 0.36-1.73 $\mathrm{p}=0.559)$, el de grasa $(\mathrm{OR}=0.846 \mathrm{IC} 95 \%$ 0.48-1.46 $\mathrm{p}=0.551)$, o el consumo de carbohidratos (OR=0.914 IC $95 \%$ 0.58-1.43 $\mathrm{p}=0.696$ ).

Para establecer relación entre las actividades físicas y éstas con la clasificación nutricional determinada por el índice de masa corporal, se utilizó el coeficiente de correlación de Spearman, encontrándose en la población total una relación inversa entre el tiempo dedicado a ver televisión, películas de video o escuchar música de lunes a viernes y la participación en actividades físicas extracurriculares, es decir, en la medida en que disminuye la asistencia a grupos organizados se incrementa el tiempo dedicado a las actividades consideradas como sedentarias $(r=-0.488)$.

Se observó, una relación entre el tiempo dedicado a juegos por computador o videojuegos de lunes a viernes y los fines de semana; los estudiantes que más juegan durante la semana, aumentan el tiempo dedicado a estas actividades durante los fines de semana $(r=0.615)$.

En la población total, a medida que aumentan las horas dedicadas a ver televisión de lunes a vienes, se incrementan los fines de semana $(r=0.374)$, lo mismo sucede con el tiempo dedicado a los juegos por computador o video juegos $(\mathrm{r}=0.661)$.
En los niños con sobrepeso y obesidad, se encontró la misma relación ( $r=0.554)$ y en este grupo se observa que cuanto menos tiempo realizan ejercicio en una clase de educación física en el colegio, menos participan en grupos deportivos en el colegio $(r=0.336)$ o equipos deportivos extracurriculares $(r=0.338)$.

Los niños con un estado nutricional normal según el índice de masa corporal, refieren gastar más tiempo haciendo ejercicio en cada clase de educación física y se incrementa su participación en equipos deportivos organizados en el colegio ( $r=0.293)$.

En los estudiantes obesos o con sobrepeso, al relacionar el índice de masa corporal con los diferentes patrones de actividad física, no se observa correlación estadísticamente significativa; sin embargo, al relacionar el porcentaje de grasa corporal por impedancia bioeléctrica, se observa una relación inversa con el tiempo que el niño realiza actividad en una clase típica de educación física, es decir, a menor tiempo de ejercicio se incrementa el porcentaje de grasa corporal $(\mathrm{r}=-0.249)$ y a medida que hay aumento de horas dedicadas a ver televisión, películas de video o escuchar música, se incrementa el porcentaje de grasa corporal ( $\mathrm{r}=0.337)$. Esta relación no se observa al relacionar el porcentaje de grasa corporal con los otros dos métodos utilizados.

En la población total se observa que a medida que aumenta el índice subescapular/tríceps, disminuye la participación en otras actividades físicas organizadas como tomar clases de algún deporte o artes marciales $(\mathrm{r}=-0.184)$.

No se encontró correlación entre el índice cintura/ cadera y el patrón de actividad física. 


\section{Discusión}

En este estudio, se observó según el índice de masa corporal y tomando como punto de corte valores en el percentil 95 o mayores, que el 15\% de la población presenta obesidad siendo más frecuente en los niños que en las niñas.

Este es hallazgo es de gran importancia ya que diversos estudios han demostrado que niños y adolescentes con un índice mayor del percentil 95 tienen por lo menos un factor de riesgo de enfermedad cardiovascular (46).

Los hallazgos mediante la impedancia bioeléctrica reflejaron mayor porcentaje de grasa en las niñas. En los niños predomina la distribución de la grasa corporal a nivel central y en ellos se observó mayor porcentaje de grasa corporal que en las niñas mediante los pliegues tricipital y de la pantorrilla según la ecuación de Slaugter.

Este resultado coincide con lo descrito en adultos, donde se ha encontrado que la distribución de la grasa a nivel central está más asociada con las alteraciones en los niveles de lípidos en los hombres que en las mujeres y el exceso de los depósitos de grasa abdominal, expresados como aumento del índice cintura/cadera aumenta el riesgo a las enfermedades cardiovasculares (47).

La medida de la circunferencia de la cintura demostró estar asociada con la clasificación nutricional por índice de masa corporal, lo cual indica que cifras altas de circunferencia de la cintura acompañan al sobrepeso y la obesidad, lo que refuerza el uso de esta variable como indicador de riesgo. Este hallazgo coincide con lo observado en diferentes poblaciones de niños y adolescentes a nivel mundial en quienes se ha observado prevalencia de sobrepeso, obesidad y exceso de grasa abdominal (48-50). Es importante resaltar que la circunferencia de la cintura como un indicador para detectar riesgo para enfermedades cardiovasculares en los niños, no es aún aceptado universalmente y hay estudios que demuestran que el índice de masa corporal es el mejor indicador para este propósito (51).

En este estudio se observó una relación directa entre el índice de masa corporal y el porcentaje de grasa obtenido a partir de la impedancia bioeléctrica; la grasa total aumenta a medida que aumenta el índice de masa corporal.

En la población total también se observó una tendencia hacia una mayor centralización de la grasa corporal mediante la relación entre el pliegue cutáneo subescapular y el pliegue tricipital, aunque no se observaron diferencias por género. Estos resultados son similares a los observados en el estudio realizado en Argentina con 711 escolares de ambos sexos, entre los 3 y 14 años de edad aunque en esa población se encontró que en promedio las mujeres tenían mayores valores que los hombres en la relación del pliegue cutáneo subescapular y el pliegue tricipital (52).

El estilo de vida y los diversos factores relacionados como el consumo de alimentos y el patrón de actividad física, se han reconocido como predictores importantes del peso corporal. En la población estudiada se observó que el mayor porcentaje de estudiantes realiza solo 2 días a la semana ejercicio o actividad física por 20 minutos o más, no cumpliendo con el estándar propuesto. Estos hallazgos concuerdan con los de estudios realizados en diferentes poblaciones que han demostrado que en los niños escolares y adolescentes se observan niveles bajos de actividad física y ejercicio, lo cual está estrechamente relacionado con la obesidad, la obesidad central y trastornos en la insulina y los lípidos sanguíneos. $(39,40,50,52)$

En el presente estudio se pudo observar que en general los estudiantes tienen un patrón de actividad física sedentario. Un alto porcentaje de ellos dedica por lo menos de 1 a 2 horas diarias a ver televisión, reportándose hasta 4 horas por día. Se aumenta el tiempo que ven televisión o videos y el número de estudiantes dedicados a esta actividad los fines de semana. Este hallazgo coincide con lo descrito en una población de niños chilenos de 8 a 10 años de edad en quienes se encontró un estilo de vida sedentario, con incremento en el número de horas de televisión los días festivos (29).

Los niños de este estudio dedican más tiempo a los videojuegos o juegos por computador que las niñas, en quienes se observó que un alto porcentaje refiere no dedicar tiempo a estos juegos, ni en semana, ni los fines de semana. La mayoría de estudiantes refiere no participar en equipos deportivos fuera del colegio o en otras actividades físicas organizadas como tenis, baile, gimnasia o artes marciales. Los estudiantes con 
sobrepeso y obesidad tienen poca participación en las actividades físicas organizadas tanto en el colegio como extracurricularmente. Al buscar asociación entre el predominio de grasa a nivel central y el patrón de actividad física, se encontró que el factor que está asociado al aumento en el índice cintura/cadera es el tiempo dedicado a los juegos de video o por computador los fines de semana.

En la población total de estudiantes se observó mayor consumo de energía y de carbohidratos en las niñas que en los niños, lo que no se encontró en el grupo de estudiantes con sobrepeso y obesidad. La distribución calórica entre los macronutrientes a partir del consumo diario de alimentos demostró estar dentro de los estándares recomendados.

Desde el punto de vista de los factores nutricionales y el patrón de actividad física que se han descrito como asociados para la enfermedad cardiovascular en estudios previos, se observa en esta población estudiada la presencia de sobrepeso y obesidad, la distribución abdominal de la grasa corporal principalmente en los niños y poca actividad física vigorosa por parte de los estudiantes. Diversos estudios han demostrado que existe asociación entre el número de horas destinadas a la televisión y otras actividades sedentarias, la prevalencia de obesidad y cambios significativos en los hábitos de alimentación, patrones de sueño y el rendimiento académico (53).

\section{Conclusiones y recomendaciones}

Con base en el índice de masa corporal, la prevalencia de sobrepeso en esta población escolar es de $22.4 \%$ y de obesidad de $15.3 \%$ y la prevalencia de obesidad es mayor en los niños que en las niñas, diferencia que es estadísticamente significativa.

El índice de cintura/cadera demostró que la distribución de la grasa a nivel superior del cuerpo es más frecuente en los niños que en las niñas. En las niñas se observó mayor porcentaje de grasa corporal mediante bioimpedancia bioeléctrica. Estas diferencias son estadísticamente significativas.

El porcentaje de grasa corporal obtenido por impedancia bioeléctrica y a partir de los pliegues cutáneos según las ecuaciones de Slaughter, demostraron estadísticamente una relación directa con el índice de masa corporal: a medida que aumenta el índice de masa corporal, aumenta el porcentaje de grasa corporal.

El análisis del consumo de alimentos demostró que las niñas consumen mayor cantidad de energía total y de carbohidratos, pero no se observó una diferencia estadísticamente significativa al hacer comparación del consumo de energía y nutrientes entre los niños normales y los que presentaron sobrepeso y obesidad.

La mayoría de los estudiantes reporta mayor dedicación a las actividades sedentarias como ver televisión y jugar con videojuegos o el computador, que a actividades deportivas en el colegio o en grupos organizados extracurriculares. La OMS recomienda por lo menos 60 minutos diarios de ejercicio físico para los niños escolares y en esta población se observó que sólo dos días a la semana, los estudiantes realizan en el colegio una actividad física por 20 minutos o más.

La clasificación nutricional por el índice de masa corporal no se correlacionó con la actividad física; sin embargo, el porcentaje de grasa corporal hallado por impedancia bioeléctrica, demostró que en la medida en que el estudiante realiza menos ejercicio en las clases de educación física del colegio y dedica más tiempo a ver televisión se incrementa el porcentaje de grasa corporal.

La presencia de sobrepeso y obesidad está asociada con las medidas de la circunferencia de la cintura y el porcentaje de grasa determinado por el método de impedancia bioeléctrica.

Ha sido ampliamente descrita la relación que existe entre el sobrepeso y la obesidad en niños y adolescentes y la presencia de hipertensión y complicaciones vasculares en etapas posteriores de la vida, por lo que es necesario abordar el problema de exceso de peso a edades tempranas desde una perspectiva de salud pública. Además, hay que tener presente que el exceso de peso contribuye también en la aparición de trastornos ortopédicos, complicaciones respiratorias, inmunológicas y gástricas, así como alteraciones en la conducta, pérdida de la autoestima y problemas relacionados con el rendimiento escolar. La detección precoz constituye un elemento importante para la prevención de la morbilidad posteriormente durante la edad adulta y requiere de un enfoque multidisciplinario dirigido a modificar los factores que orientan los estilos de vida. 
Con frecuencia no se ve a la obesidad como una enfermedad, sin embargo, su diagnostico y tratamiento y mejor aún, su prevención, son la clave para evitar enfermedades que resultan costosas tanto en salud como en dinero. Como ya se ha dicho, la diabetes, la hipertensión y otras enfermedades cardiovasculares secundarias a concentraciones elevadas de lípidos, ocupan no solamente los primeros lugares como motivo de consulta sino también la causa de muerte, incluso de pérdida de vidas prematuras de jóvenes en países desarrollados y en desarrollo. En este sentido, los profesionales y las instituciones de salud deben ser proactivos para dar respuesta a las necesidades de los niños y los jóvenes e incluir programas específicos orientados a la prevención y la promoción de la salud para estos grupos de personas y de esta manera hacer intervenciones en forma precoz y oportuna $(54,55)$.

Es importante reconocer que la prevención de la obesidad requiere del esfuerzo conjunto entre agentes gubernamentales, la industria, el comercio y los medios de comunicación, que desempeñan un papel importante en la promoción de alimentación saludable y los niveles adecuados de actividad física.

El reconocer que el ejercicio físico y la práctica deportiva regular junto con los adecuados hábitos de alimentación son importantes en la prevención de enfermedades crónicas frecuentes, como las cardiovasculares, motiva para la intervención con programas específicos orientados a diferentes grupos de personas con diversos estilos de vida, lo que permitirá el avance en el campo de la salud pública con beneficios para la sociedad.

En este estudio, el consumo de energía y nutrientes no mostró una diferencia estadísticamente significativa entre los estudiantes con estado nutricional normal de los que presentan sobrepeso y obesidad. Se recomienda en estudios similares, considerar más de un día para el registro del consumo de alimentos y de esta forma saber si la ingestión habitual de los escolares cambia y en qué proporción durante la semana y los fines de semana, además de considerar diferentes estratos socioeconómicos de la población escolar.

Una de las limitaciones que puede tener este estudio, es el sesgo de la información en cuanto al consumo de alimentos y el patrón de actividad física, datos obtenidos por reporte de padres de familia y el recordatorio de 24 horas realizado directamente a los niños, dificultad que se describe en la literatura con respecto a estos métodos de recolección de datos.

Es importante recordar que los sujetos de este estudio pertenecen a los estratos 3 y 4 por lo que los resultados no son extrapolables a todo el espectro socioeconómico de la población colombiana.

\section{Referencias bibliográficas}

1. Serra L, Aranceta J, Mataix J. Nutrición y Salud Pública. Métodos, bases científicas y aplicaciones. Madrid: Masson, S.A; 1995

2. Tojo R., Leis R. La obesidad en la infancia y la adolescencia. En: Moreno B., Charro A. Nutricion, actividad fisica y prevención de la obesidad. Estrategia NAOS. Madrid: Editorial Médica Panamericanal; 2007. p. 69-112.

3. Brown J. Nutrición en las diferentes etapas de la vida. Segunda edición. Mexico: McGraw Hill; 2006.

4. FAO: Perfiles nutricionales por países. Roma, 2001. Disponible en http://www.fao.org.co

5. De Onis M, Habicht JP. Antropometric reference data for international use: recommendation from a World Health Organization Expert Committee. Am J Clin Nutr. 1996; 64: 650-658

6. Ministerio de Protección Social, Instituto Colombiano de Bienestar Familiar, Instituto Nacional de Salud. Encuesta Nacional de la Situación Nutricional en Colombia 2010 ENSIN. Bogota: Oficina Asesora de Comunicaciones y Atención al Ciudadano ICBF; 2011. P. 1-509.

7. Daza $\mathrm{CH}$. Nutrición infantil y rendimiento escolar. Colombia Médica. 1997; 28(2): 92-98.

8. De Onis M, Blossner M. Prevalence and trends of overweight among preschool children in developing countries. Am J Clin Nutr. 2000; 72: 1032-9.

9. World Health Organization. Reducing Risk, Promoting Healthy Life. The World Health Report. WHO Geneva 2002. P. 1-248.

10. Raitakari OT; Juonala M; Kähönen M; Taittonen L. et al. Cardiovascular Risk Factors in Childhood and Carotid Artery Intima-media Thiskness in Adulthool. JAMA. 2003; 290 (17): 2277-2283.

11. McGill HC, McMahan CA, Zieske AW, Malcom GT, et al. Effects of nonlipid risk factors on atherosclerosis in youth with a favorable lipoprotein profile. JAMA. 2001; 103(11): 1546.

12. Health EM, Coleman KJ. Evaluation of the institutionalization of the coordinated approach to child health in a US/Mexico border community. Health Education and Behavior. 2003; 29(4): 157-164.

13. Kender S, Hoelsher D, Barroso C, Walker J, et al. The CATCH Kids Club: a pilot after-school study for improving elementary students' nutrition and physical activity. Public Health Nutrition. 2005; 8(2): 133-140.

14. World Health Organization. The world health report 2002. Reducing risks, promoting healthy life. Geneva; World Health Organization, 2002

15. Biesalski HK, Grima P. Nutrición. Texto y Atlas. Madrid: Editorial Médica Panamericana; 2007. p.380.

16. Heymsfield SB, Lohman TG, Wang Z, Going SB. Composición Corporal. $2^{\circ}$ ed. México: Mc Graw Hill; 2005. p. 521.

17. Kiess W, Marcus C, Wabitsch M. Obesity in childhood and adolescence. pediatric and adolescent medicine. Farmington: Karger Publishers; 2004. 
18. Freedmam DS, Khan LK, Serdura MK, et al. Inter-relationships among childhood BMI, childhood heigt, and adult obesity: the Bogalusa Heart Study. International Journal of Obesity. 2004; 28: 10-16

19. Sardinha LB, Teixeira PJ. Medición de la adiposidad y distribución de la grasa en relación con la salud. En: Heymsfield SB. et al. Composición corporal. México: Mc Graw Hill; 2005. p. $177-202$

20. Sopher A, Shen W, Pietrobelli A. Métodos de composición corporal pediátrica. En: Heymsfield SB. et al. Composición corporal. México: Mc Graw Hill; 2005. p. 177-202.

21. Urrejola P, Hodgson MI, Icaza MG. Evaluación de la composición corporal en niñas usando impedanciometría bioeléctrica y pliegues subcutáneos. Rev. Chil. Pediatr. 2001; 72(1): 26-33.

22. Sardinha LB, Teixeira PJ. Medición de la adiposidad y distribución de la grasa en relación con la salud. En: Heymsfield SB. et al. Composición corporal. México: Mc Graw Hill; 2005. p. $177-202$.

23. Williams DP. Going S, Lohman TG. Body fatness and risk for elevated blood pressure, total cholesterol, and serum lipoprotein ratios in children and adolescents. American Journal of Public Health. 1992; 82(3): 358-363.

24. Hills AP, Lyell L, Byrne NM. An evaluation of the methodology for the assessment of body composition in children and adolescents. En: Hills AP. Body Composition Assessment in Children and Adolescents. Switzerland: Karger Publishers; 2001. 44: 1-13.

25. Margett B, Nelson M. Design Concepts in Nutricional Epidemiology. New York: Oxford University Press; 2001. p.451.

26. FAO. Realización de encuestas nutricionales a pequeña escala. Manual de campo. Nutrición y Agricultura. 1995; 5: 184.

27. Aranceta J, Foz M, Gil B., et al. Obesidad y riesgo cardiovascular. Estudio DORICA. Madrid: Editorial Médica Panamericanal; 2004. p. 166.

28. Duke J, Huhman M, Heitzler C. Physical activity levels among children aged 9-13 years. United States 2003. MMWR. 2003; 52 (33): 785-788. Disponible en http://www.cdc.gov

29. Gattas V, Barrera G, Riumalló J, Uauy R. Actividad física en escolares chilenos normales y de baja talla. Rev. Chil. Pediatr. 1996; 67(5): 212-218.

30. Aranceta J, Foz M, Gil B, et al. Obesidad y riesgo cardiovascular. Estudio DORICA II. Madrid: Editorial Médica Panamericanal; 2007. p. 190.

31. Peña M, Bacallao J, editores. La obesidad en la pobreza: un nuevo reto para la salud pública. Washington: Organización $\mathrm{Pa}$ namericana de la Salud: Publicación científica No. 576, 2000.

32. Gracia B, Plata C, Pradilla A. Antropometría por edad, género y estrato socioeconómico de la población escolarizada de la zona urbana de Cali. Colombia Médica. 2003; 34(2): 61-68.

33. Díaz L, Gómez M. Situación Nutricional de los niños y niñas escolarizadas en el sector oficial de Bucaramanga, 2002. Med UNAB. 2003; 16 (17): 70-75

34. Freedman DS, Kahn H, Mei Z. et al. Relation of body mass index and waist-to-height ratio to cardiovascular disease risk factors in children and adolescents: the Bogalusa Heart Study. Am J Clin Nutr. 2007; 86: 33-40.

35. Hoelscher D, Day RS, Lee ES, Frankowski R. et al. Measuring the Prevalence of Overweight in Texas Schoolchildren. American Journal of Public Health. 2004; 94 (6): 1002.

36. Pérula L, Herrera E, Miguel MD, Lora N. Hábitos alimentarios de los escolares de una zona básica de salud de Córdoba. Rev Esp Salud Pública. 1998; 72(2): 147-50.
37. Uscátegui R, Alvarez M, Laguado I, et al. Cardiovascular risk factors in children and teenagers aged 6-18 years old from Medellín (Colombia). An. Pediatr (Barc.). 2003; 411-7.

38. Atalah E, Urteaga C, Rebolledo A, et al. Patrones alimentarios y de actividad física en escolares de la Región de Aysén. Rev. Chil. Pediatr. 1999; 70(6): 483-490.

39. Vásquez F, Salazar G, Rodríguez M, Andrade M. Comparación entre la ingesta alimentaria de preescolares obesos y eutróficos asistentes a jardines infantiles de Junji de la zona Oriente de Santiago. Archivos Latinoamericanos de Nutrición. 2007; 57(4): 343-348.

40. Poletti O, Barrios L. Obesidad e hipertensión arterial en escolares de la ciudad de Corrientes, Argentina. Arch Argent Pediatr. 2007; 105(4): 293-298.

41. Ministerio de Educación Nacional. Educación Física, Recreación y Deporte. Lineamientos Curriculares. Bogotá: 2005. Disponible en: http://www.mineducacion.gov.co

42. Centers for Disease Control and Prevention. Guidelines for school and community programs promoting lifelong physical activity. MMWR. 2001; 46(7): 1-42.

43. Bray G, Bouchard C. Handbook of obesity - Clinical Applications. Tercera edición. New York : Ed. Informa Healthcare; 2008.

44. Pardo J, Marín C, Pinzón L. Tabla de intercambios de alimentos para uso en pediatría [trabajo de grado]. Bogotá: Universidad Nacional de Colombia, Facultad de Medicina, Departamento de Nutrición y Dietética; 2000.

45. Instituto Colombiano de Bienestar Familiar. Tabla de Composición de Alimentos Colombianos. Bogotá: 2005.

46. Benjumea M, Bacallao J, Dussan C. Concordancia del índice de peso para la talla con el índice de masa corporal. Rev Cubana Salud Pública. 2002; 28(2): 76-99.

47. Seidell J, Cigollini M, Charzawska J, Ellsinger B, et al. Fat distribution and gender differences in serum lipids in men and women from European communities. Atheroesclerosis. 1991; 87: 203-210.

48. Hirscheler V, Delfino A, Clemente G, Aranda C, et al. ¿Es la circunferencia de la cintura un componente del síndrome metabólico de la infancia? Arch.argent.pediatr. 2005; 103(1): 7-13.

49. Carmenate M, Marrodán M, Mesa M, González M, Alba J. Obesidad y circunferencia de la cintura en adolescentes madrileños. Rev Cubana Salud Pública. 2007; 33(3): 10-19.

50. Cabrera A, Damiani A, Chiong D, Quintero M, Fernández L. Relación entre los lípidos séricos y la distribución de grasa corporal en un grupo de niños obesos. Revista Cubana Aliment Nutr. 1996; 10(2): 10-6.

51. Garnett S, Bauer L, Srinivasan S, Lee J, Cowell C. Body mass index and waist circumference in midchildhood and adverse cardiovascular disease risk clustering in adolescence. Am J clin Nutrition. 2007; 86: 549-55.

52. Orden A, Torres M, Luis M, Cesan M, Quintero F, Oyhenart E. Evaluación del estado nutricional en escolares de bajos recursos socioeconómicos en el contexto de la transición nutricional. Arch.argent.pediatr. 2005; 103(3): 205-211.

53. Temple J, Giacomelli A, Kent K, Roemmich J, Epstein L. Televisión watching increases motivated responding for food and energy intake in children. Am J clin Nutrition. 2007; 85: 355-61.

54. Marcos-Daccarett N, Núñez-Rocha G, Salinas-Martínez A, Santos-Ayarszagoitia M, Decanini-Arcaute H. Obesidad como factor de riesgo para transtornos metabólicos en adolescentes mexicanos,2005. Rev. salud pública. 2007; 9(2): 180-193.

55. Martínez E, Devesa M, Bacallao J, Amador M. Índice subescapular/triceps: valores percentiles en niños y adolescentes cubanos. Arch Latinoam Nutr. 1993; 43: 199-203. 